\title{
Comparison of Treatment for Metabolic Disorders Associated with Autism:Reanalysis of Three Clinical Trials
}

OPEN ACCESS

Edited by:

Pilar Marcos,

Universidad de Castilla La Mancha Albacete, Spain

Reviewed by:

Sung Ung Kang,

School of Medicine, Johns Hopkins University, United States

Benjamin Boury-Jamot,

Centre Hospitalier Universitaire Vaudois (CHUV), Switzerland

*Correspondence:

Richard E. Frye

rfrye@phoenixchildrens.com

Specialty section:

This article was submitted to Neuroenergetics, Nutrition and Brain

Health,

a section of the journal

Frontiers in Neuroscience

Received: 02 November 2017

Accepted: 10 January 2018

Published: 12 February 2018

Citation:

Delhey LM, Tippett M, Rose S, Bennuri SC, Slattery JC, Melnyk S,

James SJ and Frye RE (2018)

Comparison of Treatment for Metabolic Disorders Associated with Autism:Reanalysis of Three Clinical

Trials. Front. Neurosci. 12:19.

doi: 10.3389/fnins.2018.00019

\author{
Leanna M. Delhey ${ }^{1,2,3}$, Marie Tippett ${ }^{1,3}$, Shannon Rose ${ }^{1,3}$, Sirish C. Bennuri 1,3, \\ John C. Slattery ${ }^{1,3}$, Stepan Melnyk ${ }^{1,3}$, S. Jill James ${ }^{1,3}$ and Richard E. Frye ${ }^{1,3,4,5 *}$ \\ ${ }^{1}$ Arkansas Children's Research Institute, Little Rock, AR, United States, ${ }^{2}$ Department of Epidemiology, Fay W. Boozman \\ College of Public Health, University of Arkansas for Medical Sciences, Little Rock, AR, United States, ${ }^{3}$ Department of \\ Pediatrics, University of Arkansas for Medical Sciences, Little Rock, AR, United States, ${ }^{4}$ University of Arizona College of \\ Medicine, Phoenix, AZ, United States, ${ }^{5}$ Phoenix Children's Hospital, Phoenix, AZ, United States
}

Autism spectrum disorder (ASD) affects about 1 in 45 individuals in the United States, yet effective treatments are yet to be defined. There is growing evidence that ASD is associated with abnormalities in several metabolic pathways, including the inter-connected folate, methylation and glutathione pathways. Several treatments that can therapeutically target these pathways have been tested in preliminary clinical trials. The combination of methylcobalamin (mB12) with low-dose folinic acid (LDFA) and sapropterin, a synthetic form of tetrahydrobiopterin $(\mathrm{BH} 4)$ have been studied in openlabel trials while high-dose folinic acid has been studied in a double-blind placebo controlled trial. All of these treatments have the potential to positively affect folate, methylation and glutathione pathways. Although the effect of mB12/LDFA and BH4 on methylation and glutathione metabolism have been examined in the open-label studies, these changes have not been compared to controls who received a placebo in order to account for the natural variation in the changes in these pathways. Furthermore, the recent study using high-dose folinic acid (HDFA) did not analyze the change in metabolism resulting from the treatment. Thus, we compared changes in methylation and glutathione metabolism and biomarkers of chronic oxidative stress as a result of these three treatments to individuals receiving placebo. In general, mB12/LDFA treatment had a significant effect on glutathione and cysteine metabolism with a medium effect size while $\mathrm{BH} 4$ had a significant effect on methylation and markers of chronic oxidative stress with a large effect size. HDFA treatment did not significantly influence biomarkers of methylation, glutathione or chronic oxidative stress. One caveat was that participants in the mB12/LDFA and $\mathrm{BH} 4$ studies had significantly worse markers of glutathione metabolism and chronic oxidative stress at baseline, respectively. Thus, the participants selected in these two clinical trials may have been those with the most severe metabolic abnormalities and most expected to respond to these treatments. Overall this study supports the notion that metabolic abnormalities in individuals with ASD may be amenable to targeted treatments and provide some insight into the mechanism of action of these treatments.

Keywords: autism, folinic Acid, glutathione, methylation, methylcobalamin, oxidative stress, tetrahydrobiopterin 


\section{INTRODUCTION}

Autism spectrum disorder (ASD) is a behaviorally defined disorder that is estimated to affect up to 1 in 45 individuals in the United States (Zablotsky et al., 2014). Although decades of research has investigated the etiology of this disorder, the etiology remains largely unknown. Epidemiological research suggests that ASD arises from complicated interactions between genetics and environment, but the precise mechanism by which this occurs remains unclear (Hallmayer et al., 2011; Sandin et al., 2014).

Over the last two decades, there has been growing evidence that ASD is associated with impairments in key fundamental physiological processes of the body (Rossignol and Frye, 2012a). Abnormalities which have been verified in multiple studies include impairments in mitochondrial function (Frye and Rossignol, 2011; Rossignol and Frye, 2012a,b), abnormalities in key nutrients and cofactors such as carnitine (Gargus and Lerner, 1997; Filipek et al., 2004; Celestino-Soper et al., 2012; Frye et al., 2013d), folate (Frye et al., 2013e), and tetrahydrobiopterin (Frye, 2010; Frye et al., 2010, 2013b) as well as oxidative stress with associated abnormalities in glutathione-mediated redox regulation (James et al., 2004, 2006, 2009b; Rose et al., 2012a,b; Frye et al., 2013a). What is hopeful about abnormalities in metabolic pathways is that metabolic abnormalities often can be corrected with well-tolerated treatments that either replace key metabolites or provide intermediates that by-pass the metabolic block (Frye and Rossignol, 2014, 2016; Niyazov et al., 2016; Delhey et al., 2017). Such treatments could substantially improve the lives of children with ASD as they target pathophysiological abnormalities, thus correcting underlying abnormalities in faulty biological processes rather than simply treating symptoms.

However, just because metabolic abnormalities are associated with a disorder such as ASD, it does not mean that they are causative or that even correcting them may have an effect. That is, an important caveat is that they could be correlational with ASD and merely an epiphenomenon rather than causational to biological processes associated with ASD symptomatology. One way of providing evidence that these metabolic abnormalities may be integral to important pathophysiological processes is to determine if correcting them will improve ASD symptoms.

The authors have investigated three treatments to correct metabolic abnormalities which have demonstrated improvement in behavior and/or metabolism, namely the combination of methylcobalamin (mB12) with low-dose folinic acid (LDFA) to treat oxidative stress in an open-label study (James et al., 2009a; Frye et al., 2013c), sapropterin (BH4) to normalize tetrahydrobiopterin metabolism in an open-label study (Frye et al., 2013b) and high-dose folinic acid (HDFA) to normalize central folate metabolism in a double-blind placebo-controlled study (Frye et al., 2016b). Figure 1A demonstrates where these agents are believed to act within four major interconnected metabolic pathways that are known to be disrupted in ASD. The current study will examine the empirical evidence supporting the metabolic targets of these treatments.

The association of dysfunction in the aforementioned pathways and the effectiveness of these aforementioned treatments have been supported by the studies from others. For example, both mutations (Nashabat et al., 2017) and polymorphisms (James et al., 2006) in the cobalamin receptor has been associated with the development of ASD and individuals with ASD have been shown to have decreased concentrations of cobalamin in the brain (Zhang et al., 2016). Interestingly, the age-dependent decrease in methionine synthase, the target of $\mathrm{mB} 12$ in the methionine pathway, may be accelerated in the ASD brain (Muratore et al., 2013). Treatment with cobalamin has been shown to be therapeutic in ASD in both case (Pineles et al., 2010; Corejova et al., 2015) and double-blind placebo-controlled (Bertoglio et al., 2010; Hendren et al., 2016) studies.

As has been summarized previously, early studies in ASD demonstrated central deficiencies in BH4 and both controlled and open-label trials demonstrate a therapeutic effect of sapropterin in children with ASD (Frye et al., 2010). More recent studies have studied patients with ASD and central BH4 deficiency (Frye, 2010) and a recent double-blind placebo-controlled trial has further verified the therapeutic effect of sapropterin (Klaiman et al., 2013). Both sapropterin and cobalamin are believed to target abnormal redox metabolism, oxidative stress and oxidative damage to protein, lipid and DNA that has been repeated documented to be associated with ASD in many studies (Frustaci et al., 2012).

Some of the first cases of cerebral folate deficiency (CFD) were identified to have features of ASD (Ramaekers et al., 2005). Further case-reports and case-series suggested that abnormalities in cerebral folate metabolism was isolated to ASD patients who were low-functioning or had several neurological abnormalities (Moretti et al., 2005, 2008; Ramaekers et al., 2007). Interestingly, these studies documented substantial improvement in ASD and neurological symptoms in these children with ASD when they were treated with HDFA. More recent studies have suggested the number of children with ASD that may manifest disruption in central folate metabolism may not be limited to those who are low-functioning or who have neurological symptoms (Frye et al., 2013e, 2016a) and that a substantial number of children with ASD may benefit from HDFA (Frye et al., 2016b). Figure 1B demonstrates the target of HDFA in the context of CFD.

In all three of these aforementioned clinical treatment studies performed by the authors, biomarkers of redox and methylation metabolism were measured. In the first two studies, changes in redox and methylation metabolism were reported but because the studies were open-label the changes in these biomarkers were not compared to a placebo group. Additionally, in the latter study, the changes in redox and methylation metabolism was not analyzed in the published paper. Since the latter study had a placebo group which had redox and methylation biomarkers measured at the beginning and end of the intervention, we use this placebo group as a control group for all three studies in order to determine if the changes in redox and methylation metabolism were significant as a result of the three interventions. 
A

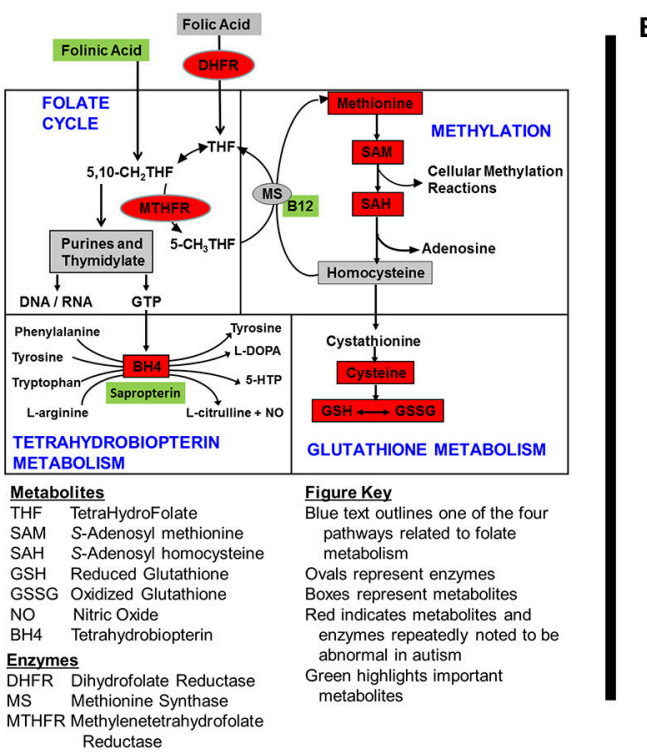

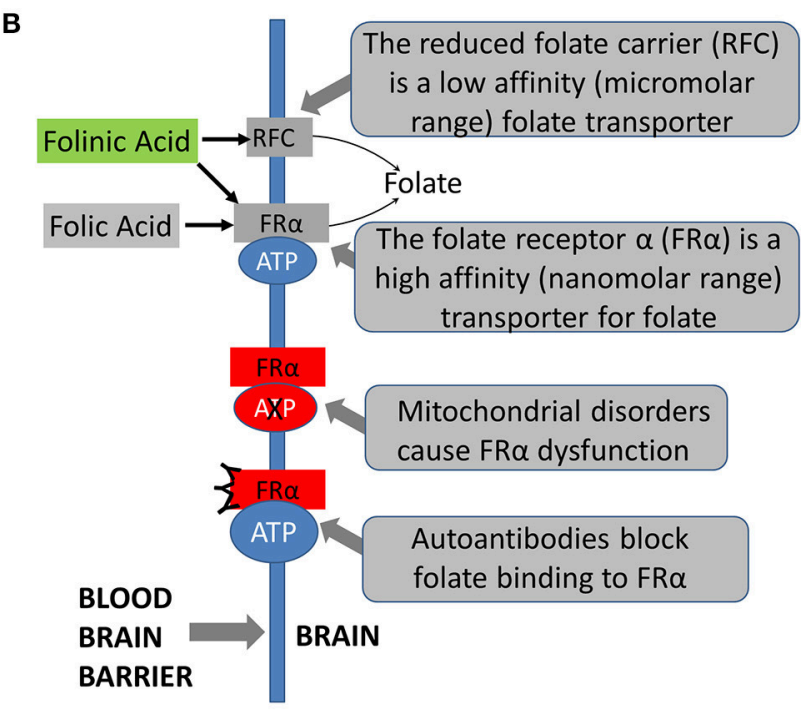

FIGURE 1 | Metabolic pathways disrupted in autism spectrum disorder (ASD). (A) The four interconnected critical folate-related metabolic pathways that manifest abnormalities in individuals with autism spectrum disorder: folate, methylation, glutathione and tetrahydrobiopterin pathways. Green boxes represent the treatments examined in this study along with their theoretical targets. Red color indicates metabolites that have been found to be abnormal in children with ASD in multiple studies from multiple laboratories. Boxes represent metabolites and ovals represent enzymes. (B) The mechanisms of folate transportation into the brain can be disrupted because of autoantibodies or mitochondrial disorders which result in dysfunction of the folate receptor alpha. Reduced folates, such as folinic acid, can use an alternative transport mechanism known as the reduced folate carrier (RFC).

\section{METHODS}

The three clinical trials have been published so we will briefly describe the methods of each. This study was carried out in accordance with the recommendations of the respective Institutional Review Boards (IRBs), described below with written informed consent from parents of subjects and assent where appropriate. Parents of all subjects gave written informed consent and participants themselves gave assent when appropriate in accordance with the Declaration of Helsinki. The protocol was approved by the IRBs named below. Table 1 provides demographics of the participants who underwent each treatment.

\section{Methylcobalamin (mB12)/Low-Dose Folinic Acid (LDFA)}

This was a prospective 12-week open-label outpatient treatment trial of subcutaneously injected methylcobalamin (mB12; $75 \mu \mathrm{g} / \mathrm{Kg}$ ) along with oral low-dose folinic acid (LDFA; $400 \mathrm{mcg}$ ) twice a day in children diagnosed with ASD who were screened for metabolic markers of poor methylation and redox metabolism (James et al., 2009a). This study was approved by the IRB of the University of Arkansas for Medical Sciences (UAMS; Little Rock AR).

\section{Tetrahydrobiopterin (BH4)}

This was a prospective 16-week open-label outpatient treatment trial of sapropterin $(20 \mathrm{mg} / \mathrm{kg})$ for 2-6 year old children with confirmed language and/or social delays, ASD and cerebrospinal fluid $\mathrm{BH}_{4}$ concentration within the normal range $(\leq 30 \mathrm{nM} / \mathrm{L})$
TABLE 1 | Demographics of the participants in the four treatment groups.

\begin{tabular}{|c|c|c|c|c|}
\hline & $N^{*}$ & Age Range & Mean Age (SD) & $\%$ Female \\
\hline $\begin{array}{l}\text { Methylcobalamin (mB12)/ } \\
\text { Low-Dose Folinic Acid } \\
\text { (LDFA) }\end{array}$ & 39 & $2 y 8 m$ to $7 y 8 m$ & $5 y 0 m(1 y 5 m)$ & $18 \%(7 / 39)$ \\
\hline Tetrahydrobiopterin (BH4) & 8 & $4 y 2 m$ to $6 y 9 m$ & $5 y 0 m(1 y 2 m)$ & $13 \%(1 / 8)$ \\
\hline $\begin{array}{l}\text { High Dose Folinic Acid } \\
\text { (HDFA) }\end{array}$ & 15 & $4 y 2 m$ to $13 y 4 m$ & $8 y 2 m(3 y 2 m)$ & $13 \%(2 / 15)$ \\
\hline Placebo & 20 & 3y $7 \mathrm{~m}$ to $12 \mathrm{y} 8 \mathrm{~m}$ & 6y $8 \mathrm{~m}(2 \mathrm{y} 10 \mathrm{~m})$ & $20 \%(4 / 20)$ \\
\hline
\end{tabular}

${ }^{*} N$ represents the number of participants that finished the treatment in the clinical trial and had a blood draw for the biomarkers under study. Thus, the number of participants are lower than the number who completed the clinical trials in most cases since blood was not always obtained from every participant at the end of the trial for various reasons, including the participant refusal.

(Frye et al., 2013b). This study was approved by the IRB of the University of Texas Health Science Center at Houston (Houston, Texas). Redox and methylation biomarkers were measured at baseline, and at 8 and 16 weeks after the start of treatment. To be consistent with the other two trials where biomarkers were measured at 12 weeks, the values for the 8 and 16 week measurements were averaged together.

\section{High Dose Folinic Acid (HDFA)}

This was a double-blind placebo-controlled outpatient treatment trial of high-dose folinic acid (HDFA) (Leucovorin Calcium $2 \mathrm{mg} / \mathrm{kg} /$ day, maximum 50mg daily) in two divided doses 
for children with ASD aged 3 to 14 years of age with language impairment (Frye et al., 2016b). This study was approved by the UAMS IRB. Of the 25 participants entering the placebo arm and the 23 participants entering the HDFA arm, 20 and 15 completed two blood draws as the second blood draw was not absolutely required for all participants.

\section{Biomarkers}

All biomarkers were collected as morning fasting samples prior to medication administration. $4 \mathrm{ml}$ of blood was collected into an EDTA-Vacutainer tube, chilled on ice and centrifuged at $1500 \times \mathrm{g}$ for 10 minutes at $4^{\circ} \mathrm{C}$. Plasma was stored at $-80^{\circ} \mathrm{C}$ and underwent HPLC with electrochemical detection within 2 weeks of collection (Melnyk et al., 1999). Selected biomarkers from the redox and methylation pathways were investigated (See Figure 1). For redox metabolism we investigated total (tGSH) and free (fGSH) reduced glutathione, oxidized glutathione (GSSG), the total and free glutathione redox ratios, total cysteine, glutamyl-cysteine and cysteinyl-glycine. For measurements of oxidative damage we measured 3-nitrotyrosine (3NT), a measure of protein oxidation, and 3-chlorotyrosine (3CT), a measure of oxidative damage due to inflammation (myeloperoxidase activity). For methylation we measured methionine, adenosine, homocysteine, S-adenosyl methionine (SAM), S-adenosyl homocysteine (SAH) and the SAM/SAH ratio, a measure of methylation capacity.

\section{Statistical Analysis}

Only participants that completed the required blood draws in the clinical trials were included in this study. No imputation for missing data was conducted and individual participants were eliminated from the analysis if laboratory data was missing. Analyses used SAS version 9.3 mixed-effects regression models to estimate the effect and effect size of the treatment (Laird and Ware, 1982). The models included the effect of time and a random intercept. The analysis is a mixed-model regression which is essentially a repeated measures ANOVA with treatment (between group) and time (within group) factors. The models tested the a priori hypothesis that the change in the metabolic biomarker was greater for participants who underwent treatment as compared with the placebo group. This effect is examined by testing the interaction between time and treatment in the model using an alpha $<0.05$. This effect is F-distributed. For each significant effect we calculate the Cohen's $d^{\prime}$ in order to demonstrate the effect size. In general a Cohen's $d^{\prime}$ effect size between 0.2 and 0.4 is small, between 0.5 and 0.7 is medium and 0.8 or above is large. In general an effect size above 0.4 is desired for any intervention.

\section{RESULTS}

\section{Baseline Differences}

Vineland adaptive behavior social, daily living and communication subscales were not significantly different across treatment groups or between treatment groups and placebo. The age of the placebo group was not significantly different than the HDFA or $\mathrm{BH} 4$ groups but was slightly but significantly higher than $\mathrm{mB} 12 / \mathrm{LDFA}$ group $\left[t_{(58)}=\right.$ 3.07, $p<0.01]$. Gender was not significantly different across groups.

Figure 2 demonstrates the metabolite baseline values for the three treatment groups and the placebo group.

Baseline values for several biomarkers were significantly different between the mB12/LDFA and placebo groups. GSSG $\left[F_{(1,57)}=6.53, p=0.01\right.$; Figure $\left.2 \mathrm{C}\right]$, total cysteine $\left[F_{(1,57)}\right.$ $=6.76, p=0.01 ;$ Figure 2F $]$, SAM $\left[F_{(1,57)}=13.67\right.$, $p<0.001$; Figure 2K $]$ and SAM/SAH $\left[F_{(1,57)}=30.79\right.$, $p<0.0001$; Figure 2M] were significantly higher in the $\mathrm{mB1} / \mathrm{LDFA}$ group as compared to the placebo group. Total GSH/GSSG $\left[F_{(1,55)}=15.31, p<0.001\right.$; Figure 2D $]$, free GSH/GSSG $\left[F_{(1,57)}=15.96, p<0.001\right.$; Figure 2E $]$, Adenosine $\left[F_{(1,57)}=15.11, p<0.001\right.$; Figure 20], Cysteinyl-Glycine $\left[F_{(1,56)}=9.02, p<0.01\right.$; Figure $\left.2 \mathrm{H}\right]$ and $\mathrm{SAH}\left[F_{(1,57)}=\right.$ 12.16, $p<0.001$; Figure $2 \mathrm{~K}$ ] were significantly lower in the $\mathrm{mB12} / \mathrm{LDFA}$ group as compared to the placebo group at baseline.

Baseline values for several biomarkers were significantly different between the $\mathrm{BH} 4$ and placebo groups. 3NT $\left[F_{(1,26)}\right.$ $=11.75, p<0.01$; Figure 2I $]$ and 3CT $\left[F_{(1,26)}=17.07, p<\right.$ 0.001; Figure 2J] where higher in the $\mathrm{BH} 4$ group as compared to the placebo group while free GSH $\left[F_{(1,26)}=7.04, p=0.01\right.$; Figure 2B], Adenosine $\left[F_{(1,26)}=8.72, p<0.01\right.$; Figure 2O] and Cysteinyl-Glycine $\left[F_{(1,26)}=5.93, p<0.05\right.$; Figure $\left.2 \mathbf{H}\right]$ were lower in the $\mathrm{BH} 4$ group as compared to the placebo group.

For the HDFA group, only free GSH/GSSG $\left[F_{(1,33)}=4.22\right.$, $p<0.05$; Figure 2E] was significantly higher than the placebo group at baseline.

\section{The Effect of Treatments on Glutathione Metabolism}

The combination of $\mathrm{mB} 12 / \mathrm{LDFA}$ significantly increased total GSH $\left[F_{(1,56)}=4.23, p<0.05\right.$; Cohen's $d^{\prime}=0.55$; Figure 3A $]$, total GSH/GSSG ratio $\left[F_{(1,54)}=5.76, p<0.05\right.$; Cohen's $d^{\prime}$ $=0.65$; Figure 3D $]$ and the free GSH/GSSG ratio $\left[F_{(1,56)}=\right.$ 4.58, $p<0.05$; Cohen's $d^{\prime}=0.57$; Figure 3E]. Although the combination of $\mathrm{mB1}$ /LDFA decreased GSSG (Figure 3C) and increased free GSH (Figure 3B) these differences did not reach significance. BH4 treatment resulted in changes in glutathione consistent with the combination of $\mathrm{mB} 12$ /LDFA treatment but only the free GSH/GSSG ratio $\left[F_{(1,26)}=7.36, p=0.01\right.$; Cohen's $d^{\prime}=1.06$; Figure $\left.3 \mathrm{E}\right]$ was statistically significant. HDFA treatment had a negligible effect on glutathione metabolism.

\section{The Effect of Treatments on Cysteine Metabolism}

The combination of mB12/LDFA significantly increased total Cysteine $\left[F_{(1,57)}=6.26, p=0.01\right.$; Cohen's $d^{\prime}$ $=0.66 ;$ Figure $3 \mathbf{F}]$ and Cysteinyl-Glycine $\left[F_{(1,56)}=\right.$ 

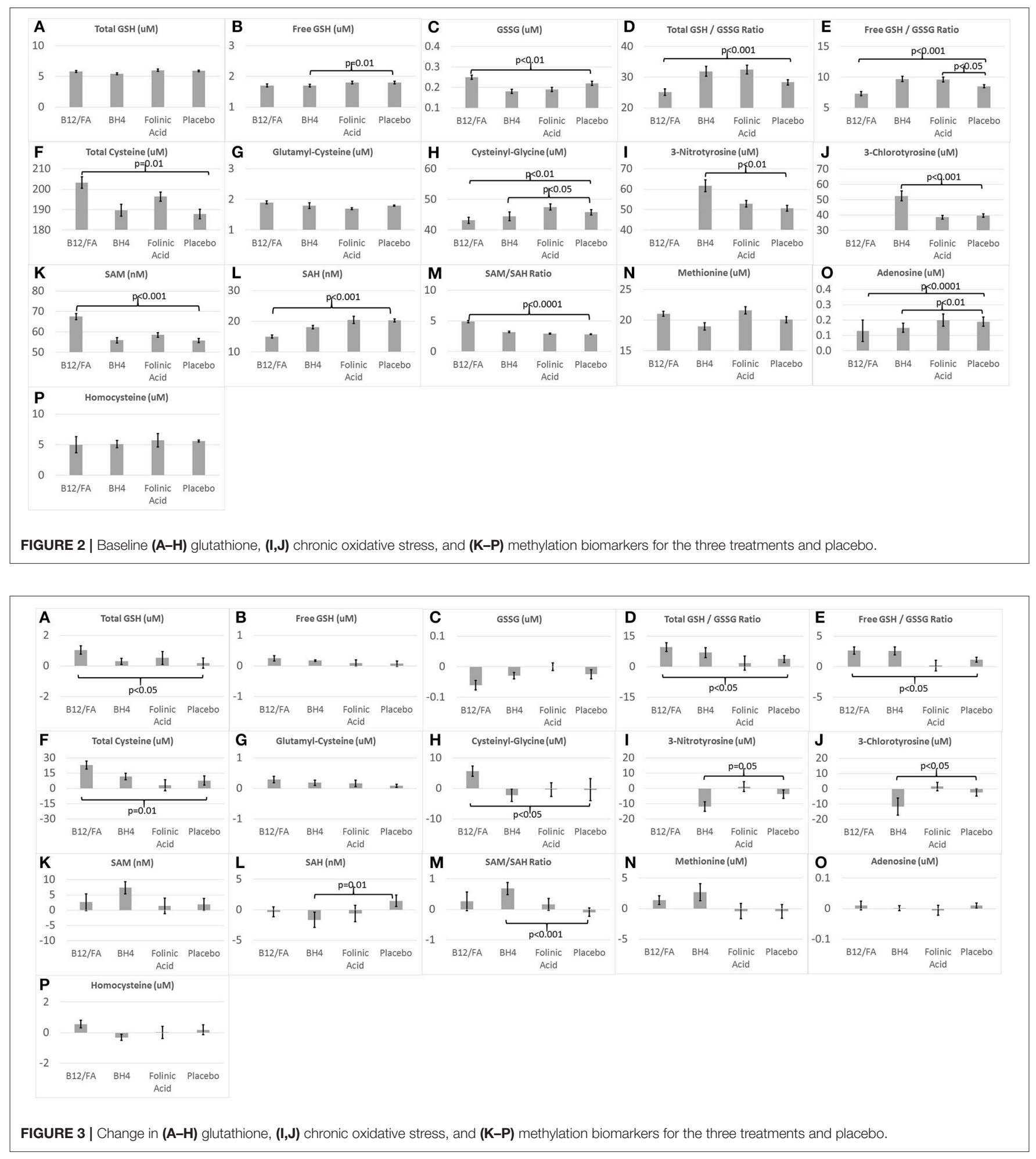

5.45, $p<0.05$; Cohen's $d^{\prime}=0.62$; Figure 3H] but did not statistically significantly change Glutamyl-Cysteine (Figure 3G) although the change was in the expected direction. BH4 and HDFA did not demonstrate effects on cysteine metabolism.

\section{The Effect of Treatments on Chronic Oxidative Stress and Inflammation}

$\mathrm{BH} 4$ treatment significant reduced $3 \mathrm{NT}\left[F_{(1,26)}=4.02, p\right.$ $=0.05 ;$ Cohen's $d^{\prime}=0.78 ;$ Figure $\left.3 \mathrm{I}\right]$ and $3 \mathrm{CT}\left[F_{(1,26)}\right.$ $=5.85$, Cohen's $d^{\prime}=0.94 ; p<0.05$; Figure 3J]. HDFA 
did not appear to have any effect on these biomarkers and these biomarkers were not examined in the mB12/LDFA study.

\section{The Effect of Treatments on Methylation Metabolism}

$\mathrm{BH} 4$ treatment resulted in a statistically significant decreased in $\mathrm{SAH}\left[F_{(1,26)}=6.95, p=0.01\right.$; Cohen's $d^{\prime}=1.03$; Figure $3 \mathrm{~L}]$ and increase in the SAM/SAH ratio $\left[F_{(1,26)}=\right.$ 14.17, $p<0.001$; Cohen's $d^{\prime}=1.47$; Figure 3M]. BH4 treatment did increase SAM but the difference was only borderline statistically significant $\left[F_{(1,26)}=3.03, p=0.09\right.$; Cohen's $d^{\prime}=0.68$; Figure $\left.3 \mathrm{~K}\right] . \mathrm{mB1} / \mathrm{LDFA}$ and HDFA did not significantly change SAM, SAH or the SAM/SAH ratio. $\mathrm{BH} 4$ treatment resulted in an increase in methionine but this increase was only borderline significant $\left[F_{(1,26)}=3.91, p\right.$ $=0.06$; Cohen's $d^{\prime}=0.78$; Figure 3N]. Neither mB12/LDFA nor HDFA had a significant effect on methionine. None of the treatments appeared to alter adenosine or homocysteine (Figures 30,P).

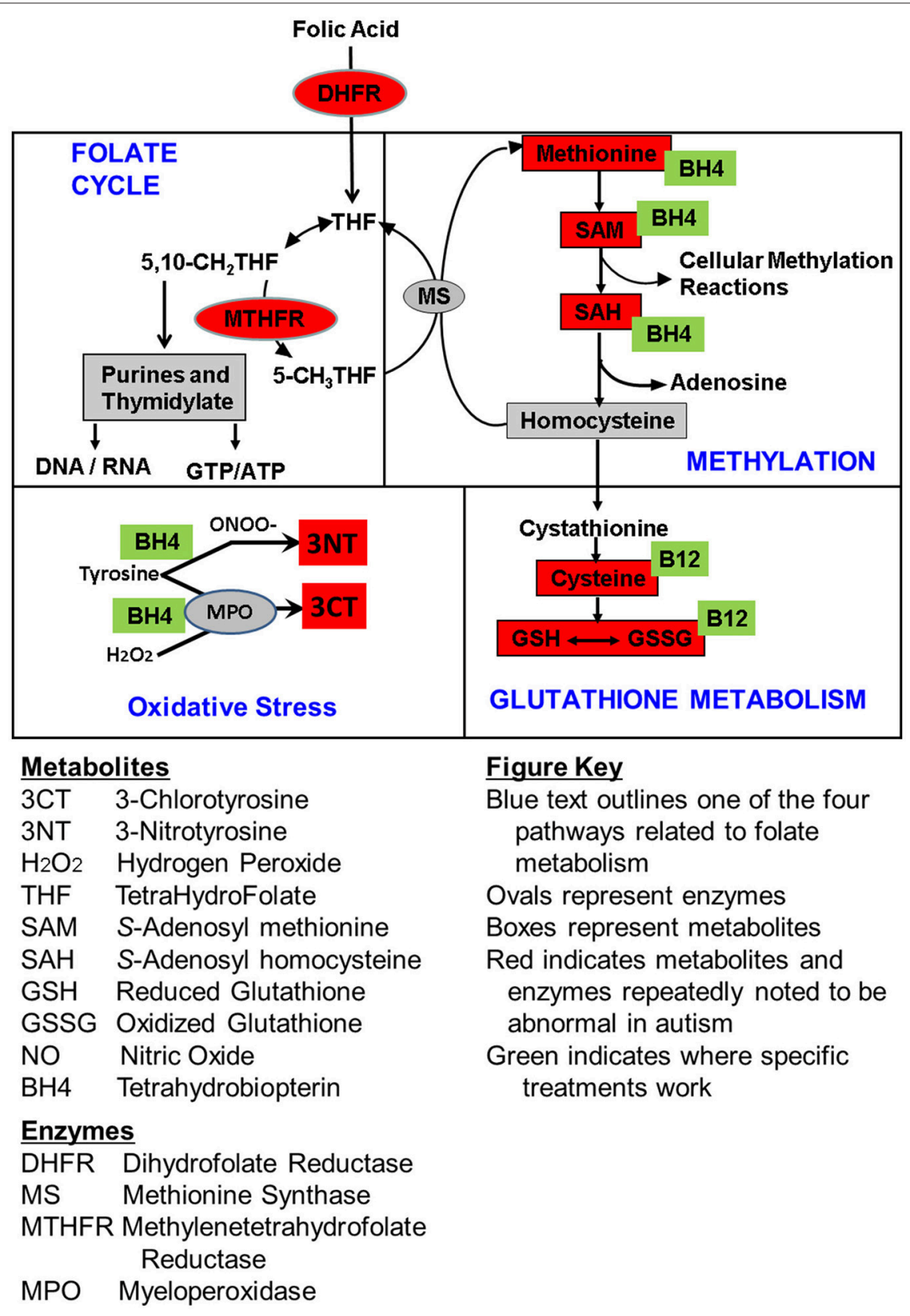

FIGURE 4 | Summary of the empirically determined metabolic targets of treatments evaluated in this study. Green boxes represent the treatments examined in this study. Red color indicates metabolites that have been found to be abnormal in children with ASD in multiple studies from multiple laboratories. Boxes represent metabolites and ovals represent enzymes. 


\section{DISCUSSION}

In this study we examined three clinical treatments that have the potential to influence methylation and redox regulation pathways as well as positively affect oxidative stress. Children with ASD were treated with one of these three treatments or placebo and the effect of each treatment over an equivalent 12 week period was compared to the effect of placebo over the same time period. These treatments include mB12/LDFA, BH4 and HDFA. Figure 4 summarizes the metabolites affected by these treatments as well as the pathway for these metabolites. In general $\mathrm{mB} 12 / \mathrm{LDFA}$ has a significant influence on redox regulation with medium effect sizes, particularly in the role of positively affecting glutathione metabolism and its precursors, whereas BH4 primarily has a positive effect on markers of oxidative damage as well as methylation metabolism with large effect sizes. Somewhat surprisingly HDFA by itself does not appear to have a major significant effect on redox regulation or methylation pathways possibly because children were not pre-screened for low baseline GSH/GSSG. This provides some empirical evidence into the mechanism of action of these treatments beyond theoretical considerations.

One limitation of examining these clinical trial data in this way is that the ASD population that received the placebo, although similar in development, age and gender, for various reasons, had some differences baseline metabolic characteristics which could affect the effectiveness of the treatment being studied. In some studies, this was by design. For example, the open-label mB12/LDFA study selected individuals with poor glutathione and methylation status to potentiate the ability of the treatment to change these parameters (James et al., 2009a). Interestingly, only glutathione and cysteine metabolism were statistically significantly improved in the mB12/LDFA trial, both with a medium effect size. When compared to the placebo group, which was unselected for metabolic abnormalities, the participants in the mB12/LDFA group demonstrated a more favorable methylation capacity, suggest that they were less severe than what was expected by implementing the preselection or that the pathway was up-regulated under conditions of oxidative stress. The mB12/LDFA group did demonstrate less favorable glutathione metabolism than the placebo group at baseline, suggest that the pre-selection process was useful for obtaining children with the worse glutathione metabolism abnormalities. This study confirms that the group of children with ASD and glutathione abnormalities were indeed improved with $\mathrm{mB} 12 / \mathrm{LDFA}$ treatment as previously hypothesized and supported by the behavioral data (Frye et al., 2013c). The medium effect size suggests that this is an acceptable therapeutic intervention.

\section{REFERENCES}

Bertoglio, K., Jill James, S., Deprey, L., Brule, N., and Hendren, R. L. (2010). Pilot study of the effect of methyl B12 treatment on behavioral and biomarker measures in children with autism. J. Altern. complement. Med. 16, 555-560. doi: $10.1089 /$ acm.2009.0177
Like the participants in the mB12/LDFA study, participants in the BH4 study demonstrated some less favorable biomarkers at baseline, but these biomarkers were related to chronic oxidative stress and inflammation. These biomarkers related to chronic oxidative stress and inflammation were significantly abnormal at baseline in the $\mathrm{BH} 4$ treatment participants as compared to those who received placebo. Thus, the data from this study support the notion that $\mathrm{BH} 4$ can improve oxidative damage and methylation abnormalities in children with ASD with a large effect size, with the caveat that this effect may be more pronounced in those with the more severe abnormalities at baseline.

The fact that folinic acid, even at high dose, did not change metabolic markers of methylation and/or glutathione metabolism unless it was combined with $\mathrm{mB} 12$ is an important finding as it has direct implication for treatment of inter-related but distinct metabolic disorders associated with ASD. HDFA treatment targets the blockage in the transportation of folate into the brain through the folate receptor alpha caused by blocking or binding autoantibodies (Frye et al., 2013e, 2016a,b, 2017a,b) and is not intended to target methylation or glutathione metabolism directly. These data support the notion that folinic acid needs to be combined with $\mathrm{mB} 12$ in order to improve glutathione metabolism.

Overall, this study provides some insight to the potential benefits of safe and well-tolerated therapies that target specific metabolism abnormalities associated with ASD. A better understanding of these physiological abnormalities could lead to the discovery of novel pathways for treatments of potentially many neurodevelopmental disorders and other neurological disorders involving abnormalities in these metabolic pathways.

\section{AUTHOR CONTRIBUTIONS}

LD, MT, and JS Collected that data and samples. SR, SB, and SM processed the samples and performed the assays. RF and SJ designed the studies. RF drafted the manuscript. All authors read and agreed with the manuscript submission.

\section{FUNDING}

This research was supported by the Arkansas Biosciences Institute (Little Rock, AR, USA), The Jonty Foundation (St Paul, MN), The Autism Research Institute (San Diego, CA), the Gupta Family Foundation (Atherton, CA), The Jane Bostford Johnson Foundation (New York, NY) and the Jager Family Foundation (Chicago, IL).

Celestino-Soper, P. B., Violante, S., Crawford, E. L., Luo, R., Lionel, A. C., Delaby, E., et al. (2012). A common X-linked inborn error of carnitine biosynthesis may be a risk factor for nondysmorphic autism. Proc. Natl. Acad. Sci. U.S.A. 109, 7974-7981. doi: 10.1073/pnas.1120210109

Corejova, A., Janosikova, D., Pospisilova, V., Rauova, D., Kyselovic, J., and Hrabovska, A. (2015). Cessation of Nocturnal Enuresis after Intervention with 
Methylcobalamin in an 18-Year-Old Patient with Autism. J. Child Adolesc. Psychopharmacol. 25, 821-823. doi: 10.1089/cap.2014.0023

Delhey, L. M., Nur Kilinc, E., Yin, L., Slattery, J. C., Tippett, M. L., Rose, S., et al. (2017). The effect of mitochondrial supplements on mitochondrial activity in children with autism spectrum disorder. J. Clin. Med. 6:18. doi: $10.3390 /$ jcm 6020018

Filipek, P. A., Juranek, J., Nguyen, M. T., Cummings, C., and Gargus, J. J. (2004). Relative carnitine deficiency in autism. J. Autism Dev. Disord. 34, 615-623. doi: 10.1007/s10803-004-5283-1

Frustaci, A., Neri, M., Cesario, A., Adams, J. B., Domenici, E., Dalla Bernardina, B., et al. (2012). Oxidative stress-related biomarkers in autism: systematic review and meta-analyses. Free Radical Biol. Med. 52, 2128-2141. doi: $10.1016 /$ j.freeradbiomed.2012.03.011

Frye, R. E., and Rossignol, D. A. (2011). Mitochondrial dysfunction can connect the diverse medical symptoms associated with autism spectrum disorders. Pediatr. Res. 69(5 Pt 2), 41R-47R. doi: 10.1203/PDR.0b013e318212f16b

Frye, R. E., DeLatorre, R., Taylor, H. B., Slattery, J., Melnyk, S., Chowdhury, N., et al. (2013a). Metabolic effects of sapropterin treatment in autism spectrum disorder: a preliminary study. Transl. Psychiatry 3:e237. doi: 10.1038/tp.20 13.14

Frye, R. E., Delatorre, R., Taylor, H., Slattery, J., Melnyk, S., Chowdhury, N., et al. (2013b). Redox metabolism abnormalities in autistic children associated with mitochondrial disease. Transl. Psychiatry 3:e273. doi: 10.1038/tp.2013.51

Frye, R. E., Melnyk, S., Fuchs, G., Reid, T., Jernigan, S., Pavliv, O., et al. (2013c). Effectiveness of methylcobalamin and folinic Acid treatment on adaptive behavior in children with autistic disorder is related to glutathione redox status. Autism Res. Treat. 2013:609705. doi: 10.1155/2013/609705

Frye, R. E., Melnyk, S., and Macfabe, D. F. (2013d). Unique acyl-carnitine profiles are potential biomarkers for acquired mitochondrial disease in autism spectrum disorder. Transl. Psychiatry 3:e220. doi: 10.1038/tp.2012.143

Frye, R. E., Sequeira, J. M., Quadros, E. V., James, S. J., and Rossignol, D. A. (2013e). Cerebral folate receptor autoantibodies in autism spectrum disorder. Mol. Psychiatry 18, 369-381. doi: 10.1038/mp.2011.175

Frye, R. E., Huffman, L. C., and Elliott, G. R. (2010). Tetrahydrobiopterin as a novel therapeutic intervention for autism. Neurotherapeutics 7, 241-249. doi: 10.1016/j.nurt.2010.05.004

Frye, R. E., and Rossignol, D. A. (2014). Treatments for biomedical abnormalities associated with autism spectrum disorder. Front. Pediatr. 2, 66. doi: $10.3389 /$ fped.2014.00066

Frye, R. E., and Rossignol, D. A. (2016). Identification and Treatment of Pathophysiological Comorbidities of Autism Spectrum Disorder to Achieve Optimal Outcomes. Clin. Med. Insights Pediatr. 10, 43-56. doi: 10.4137/CMPed.S38337

Frye, R. E., Slattery, J., Delhey, L., Furgerson, B., Strickland, T., Tippett, M., et al. (2016a). Folinic acid improves verbal communication in children with autism and language impairment: a randomized double-blind placebo-controlled trial. Mol Psychiatry. doi: 10.1038/mp.2016.168. [Epub ahead of print].

Frye, R. E., Delhey, L., Slattery, J., Tippett, M., Wynne, R., Rose, S., et al. (2016b). Blocking and binding folate receptor alpha autoantibodies identify novel autism spectrum disorder subgroups. Front. Neurosci. 10:80. doi: $10.3389 /$ fnins.2016.00080

Frye, R. E., Slattery, J. C., and Quadros, E. V. (2017a). Folate metabolism abnormalities in autism: potential biomarkers. Biomark Med. doi: 10.2217/bmm-2017-0109. [Epub ahead of print].

Frye, R. E., Wynne, R., Rose, S., Slattery, J., Delhey, L., Tippett, M., et al. (2017b). Thyroid dysfunction in children with autism spectrum disorder is associated with folate receptor alpha autoimmune disorder. J. Neuroendocrinol. doi: 10.1111/jne.12461. [Epub ahead of print].

Frye, R. E. (2010). Central tetrahydrobiopterin concentration in neurodevelopmental disorders. Front. Neurosci. 4:52. doi: $10.3389 /$ fnins. 2010.00052

Gargus, J. J., and Lerner, M. A. (1997). Familial autism with primary carnitine deficiency, sudden death, hypotonia and hypochromic anemia. Am. J. Hum. Gen. 61:A98.

Hallmayer, J., Cleveland, S., Torres, A., Phillips, J., Cohen, B., Torigoe, T., et al. (2011). Genetic heritability and shared environmental factors among twin pairs with autism. Arch. Gen. Psychiatry 68, 1095-1102. doi: $10.1001 /$ archgenpsychiatry.2011.76
Hendren, R. L., James, S. J., Widjaja, F., Lawton, B., Rosenblatt, A., and Bent, S. (2016). Randomized, placebo-controlled trial of methyl B12 for children with autism. J. Child Adolesc. Psychopharmacol. 26, 774-783. doi: 10.1089/cap.2015.0159

James, S. J., Cutler, P., Melnyk, S., Jernigan, S., Janak, L., Gaylor, D. W., et al. (2004). Metabolic biomarkers of increased oxidative stress and impaired methylation capacity in children with autism. Am. J. Clin. Nutri. 80, 1611-1617.

James, S. J., Melnyk, S., Fuchs, G., Reid, T., Jernigan, S., Pavliv, O., et al. (2009b). Efficacy of methylcobalamin and folinic acid treatment on glutathione redox status in children with autism. Am. J. Clin. Nutr. 89, 425-430. doi: $10.3945 /$ ajen.2008.26615

James, S. J., Melnyk, S., Jernigan, S., Cleves, M. A., Halsted, C. H., Wong, D. H., et al. (2006). Metabolic endophenotype and related genotypes are associated with oxidative stress in children with autism. Am. J. Med. Genet. B. Neuropsychiatr. Genet. 141b, 947-56. doi: 10.1002/ajmg.b.30366

James, S. J., Rose, S., Melnyk, S., Jernigan, S., Blossom, S., Pavliv, O., et al. (2009a). Cellular and mitochondrial glutathione redox imbalance in lymphoblastoid cells derived from children with autism. FASEB 23, 2374-2383. doi: $10.1096 / \mathrm{fj} .08-128926$

Klaiman, C., Huffman, L., Masaki, L., and Elliott, G. R. (2013). Tetrahydrobiopterin as a treatment for autism spectrum disorders: a double-blind, placebocontrolled trial. J. Child Adolesc. Psychopharmacol. 23, 320-328. doi: 10.1089 /cap.2012.0127

Laird, N. M., and Ware, J. H. (1982). Random-effects models for longitudinal data. Biometrics 38, 963-974. doi: 10.2307/2529876

Melnyk, S., Pogribna, M., Pogribny, I., Hine, R. J., and James, S. J. (1999). A new HPLC method for the simultaneous determination of oxidized and reduced plasma aminothiols using coulometric electrochemical detection. J. Nutr. Biochem. 10, 490-497. doi: 10.1016/S0955-2863(99)00033-9

Moretti, P., Peters, S. U., Del Gaudio, D., Sahoo, T., Hyland, K., Bottiglieri, T., et al. (2008). Brief report: autistic symptoms, developmental regression, mental retardation, epilepsy, and dyskinesias in CNS folate deficiency. J. Autism Dev. Disord. 38, 1170-1177. doi: 10.1007/s10803-007-0492-z

Moretti, P., Sahoo, T., Hyland, K., Bottiglieri, T., Peters, S., del Gaudio, D., et al. (2005). Cerebral folate deficiency with developmental delay, autism, and response to folinic acid. Neurology 64, 1088-1090. doi: 10.1212/01.WNL.0000154641.08211.B7

Muratore, C. R., Hodgson, N. W., Trivedi, M. S., Abdolmaleky, H. M., Persico, A. M., Lintas, C., et al. (2013). Age-dependent decrease and alternative splicing of methionine synthase mRNA in human cerebral cortex and an accelerated decrease in autism. PLoS ONE 8:e56927. doi: 10.1371/journal.pone.0056927.

Nashabat, M., Maegawa, G., Nissen, P. H., Nexo, E., Al-Shamrani, H., Al-Owain, M., et al. (2017). Long-term outcome of 4 patients with transcobalamin deficiency caused by 2 Novel TCN2 Mutations. J. Pediatr. Hematol. 39, e430e436. doi: 10.1097/MPH.0000000000000857

Niyazov, D. M., Kahler, S. G., and Frye, R. E. (2016). Primary mitochondrial disease and secondary mitochondrial dysfunction: importance of distinction for diagnosis and treatment. Mol. Syndromol. 7, 122-137. doi: 10.1159/000446586

Pineles, S. L., Avery, R. A., and Liu, G. T. (2010). Vitamin B12 optic neuropathy in autism. Pediatrics 126, e967-e970. doi: 10.1542/peds.2009-2975

Ramaekers, V. T., Rothenberg, S. P., Sequeira, J. M., Opladen, T., Blau, N., Quadros, E. V., et al. (2005). Autoantibodies to folate receptors in the cerebral folate deficiency syndrome. New Engl. J. Med. 352, 1985-1991. doi: 10.1056/NEJMoa043160

Ramaekers, V. T., Blau, N., Sequeira, J. M., Nassogne, M. C., and Quadros, E. V. (2007). Folate receptor autoimmunity and cerebral folate deficiency in lowfunctioning autism with neurological deficits. Neuropediatrics 38, 276-281. doi: 10.1055/s-2008-1065354

Rose, S., Melnyk, S., Pavliv, O., Bai, S., Nick, T. G., Frye, R. E., et al. (2012a). Evidence of oxidative damage and inflammation associated with low glutathione redox status in the autism brain. Transl. Psychiatry 2:e134. doi: $10.1038 /$ tp.2012.61

Rose, S., Melnyk, S., Trusty, T. A., Pavliv, O., Seidel, L., Li, J., et al. (2012b). Intracellular and extracellular redox status and free radical generation in primary immune cells from children with autism. Autism Res. Treat. 2012:986519. doi: 10.1155/2012/986519

Rossignol, D. A., and Frye, R. E. (2012a). A review of research trends in physiological abnormalities in autism spectrum disorders: immune 
dysregulation, inflammation, oxidative stress, mitochondrial dysfunction and environmental toxicant exposures. Mol. Psychiatry 17, 389-401. doi: $10.1038 / \mathrm{mp} .2011 .165$

Rossignol, D. A., and Frye, R. E. (2012b). Mitochondrial dysfunction in autism spectrum disorders: a systematic review and meta-analysis. Mol. Psychiatry 17, 290-314. doi: 10.1038/mp.2010.136

Sandin, S., Lichtenstein, P., Kuja-Halkola, R., Larsson, H., Hultman, C. M., and Reichenberg, A. (2014). The familial risk of autism. JAMA 311, 1770-1777. doi: 10.1001/jama.2014.4144

Zablotsky, B., Black, L. I., Maenner, M. J., Schieve, L. A., and Blumberg, S. J. (2014). Estimated prevalence of autism and other developmental disabilities following questionnaire changes in the national health interview survey. Natl. Health Stat. Rep. 2015:1-20.

Zhang, Y., Hodgson, N. W., Trivedi, M. S., Abdolmaleky, H. M., Fournier, M., Cuenod, M., et al. (2016). Decreased brain levels of vitamin B12 in aging, autism and schizophrenia. PLoS ONE 11:e0146797. doi: 10.1371/journal.pone.0 146797

Conflict of Interest Statement: The authors declare that the research was conducted in the absence of any commercial or financial relationships that could be construed as a potential conflict of interest.

Copyright (c) 2018 Delhey, Tippett, Rose, Bennuri, Slattery, Melnyk, James and Frye. This is an open-access article distributed under the terms of the Creative Commons Attribution License (CC BY). The use, distribution or reproduction in other forums is permitted, provided the original author(s) and the copyright owner are credited and that the original publication in this journal is cited, in accordance with accepted academic practice. No use, distribution or reproduction is permitted which does not comply with these terms. 